\title{
Fast-ion transport in the presence of magnetic reconnection induced by sawtooth oscillations in ASDEX Upgrade
}

\author{
B. Geiger, ${ }^{1}$ M. Garcia-Munoz,${ }^{2}$ R. Dux,${ }^{1}$ F. Ryter,${ }^{1}$ G. Tardini,${ }^{1}$ L. Barrera Orte,${ }^{1}$ I.G.J. Classen, ${ }^{3}$ \\ E. Fable ${ }^{1}$ R. Fischer ${ }^{1}$ V. Igochine, ${ }^{1}$ R.M. McDermott,${ }^{1}$ and the ASDEX Upgrade Team ${ }^{1}$ \\ ${ }^{1}$ Max Planck Institute for Plasma Physics, Boltzmannstr. 2, 85748 Garching, Germany \\ ${ }^{2}$ Faculty of Physics, University of Seville, Spain \\ ${ }^{3}$ Differ, EURATOM Association, Netherlands
}

(Dated: January 31, 2014)

\begin{abstract}
The transport of beam-generated fast ions has been investigated experimentally at the ASDEX Upgrade tokamak in the presence of sawtooth crashes. After sawtooth crashes, phase space resolved fast-ion D-alpha (FIDA) measurements show a significant reduction of the central fast-ion density more than 50\% - together with an increase at larger radii. The corresponding loss of fast particles, detected by a fast-ion loss detector (FILD) outside the plasma, is marginal during the crash phase, which proves that the sawteeth internally redistribute fast ions. This is also well reproduced by modelling results in which the fast-ion redistribution is assumed to be in line with the sawtoothinduced change of the magnetic field topology. The simulation, however, underestimates the total amount of redistributed fast ions which can be explained by additional drift effects. In the time intervals between the crashes, a neo-classical fast-ion behavior is observed. Radial profiles and measured decay rates of the redistributed fast ions show that any anomalous fast-ion diffusion in between sawtooth crashes is well below $0.5 \mathrm{~m}^{2} / \mathrm{s}$.
\end{abstract}

PACS numbers: 52.55.Fa, 52.50.Gj, 52.55.Tn, 52.70.Kz

Introduction- Good confinement of fast-ions, whether generated by fusion processes or neutral beam injection (NBI), is essential in fusion devices because these suprathermal particles are responsible for plasma heating, current drive and can be critical for machine safety [1]. Mechanisms that cause radial transport of fast-particles must consequently be investigated, understood and, if possible, avoided. While weak, neoclassical fast-ion diffusion is expected in the absence of perturbations, turbulent transport [2] as well as magneto-hydrodynamic (MHD) modes could significantly redistribute fast ions [3]. One prominent MHD mode is the sawtooth instability [4] that appears periodically in the plasma center due to the magnetic reconnection of a mode at the $q=1$ surface ( $q$ is the safety factor). This mode radially redistributes heat, particles and momentum during a fast crash $\left(\sim 10^{-4} \mathrm{~s}\right)$ which is followed by a slower recovery phase. A basic, but successful approach to describe sawtooth crashes is the Kadomtsev model [5] which assumes full reconnection and a strong transport due to the evolving field lines up to the so-called mixing radius. It does, however, not take the electric fields that appear during the reconnection process into account. The latter are supposed to affect the fast-ions via $\mathbf{E x B}$ drifts if the fast-ion energy is above a given critical value [6]. An effect of sawtooth crashes on fast-ions was already observed more than 20 years ago at PLT, DIII-D, TFTR [7] and JET [8] via measurements of reduced neutron rates and in D-T experiments via modified fast alpha particle radiation profiles [9-13]. In addition, more recent measurements at TEXTOR [14] and DIII-D [15] revealed a stronger redistribution of the passing fast ions than the trapped ones as predicted by [6]. However, the fast-ion redistribution due to sawtooth crashes is still not com- pletely understood because fast-ion diagnostics are typically limited in both spatial and temporal resolution, which makes comparisons with theory difficult.

This letter presents the first detailed and time-resolved measurements of fast ions in the presence of sawtooth activity that have been carried out at the medium size tokamak ASDEX Upgrade (minor and major radii of $0.5 \mathrm{~m}$ and $1.65 \mathrm{~m}$, respectively). In particular, a newly developed and installed fast-ion D-alpha (FIDA) diagnostic [16] enables us to investigate the evolution of the confined fast ions in the plasma with $2 \mathrm{~ms}$ exposure time and $3 \mathrm{~cm}$ spatial resolution. The FIDA diagnostic analyzes strongly Doppler shifted Balmer alpha radiation $\left(\lambda_{0}=656.1 \mathrm{~nm}\right)$ from fast deuterium ions that are neutralized by charge exchange reactions along an NBI path. It uses multiple radially distributed lines of sight (LOS) with two different viewing geometries (poloidal and toroidal) to access distinct regions in the velocity space. In addition, a fast-ion loss detector (FILD [17]) allows us to monitor the fast ions that leave the plasma with a $1 \mathrm{MHz}$ bandwidth. The FILD measurements show that only very weak fast-ion losses appear during the crashes while the FIDA diagnostic clearly measures a significant internal redistribution that is even stronger than that predicted by the Kadomtsev model. Moreover, the measured radial profiles and slowing down times of the fast ions after sawtooth crashes evidence neoclassical confinement and hence show that turbulent transport effects are negligible under the investigated conditions.

Experiment- Characteristic time traces of a representative discharge with a current of $1 \mathrm{MA}$ and a magnetic field of $-2.3 \mathrm{~T}$ are displayed in figure 1 . The sawtooth crashes are clearly visible as sharp drops in the electron and ion temperatures and toroidal rotation, measured in 


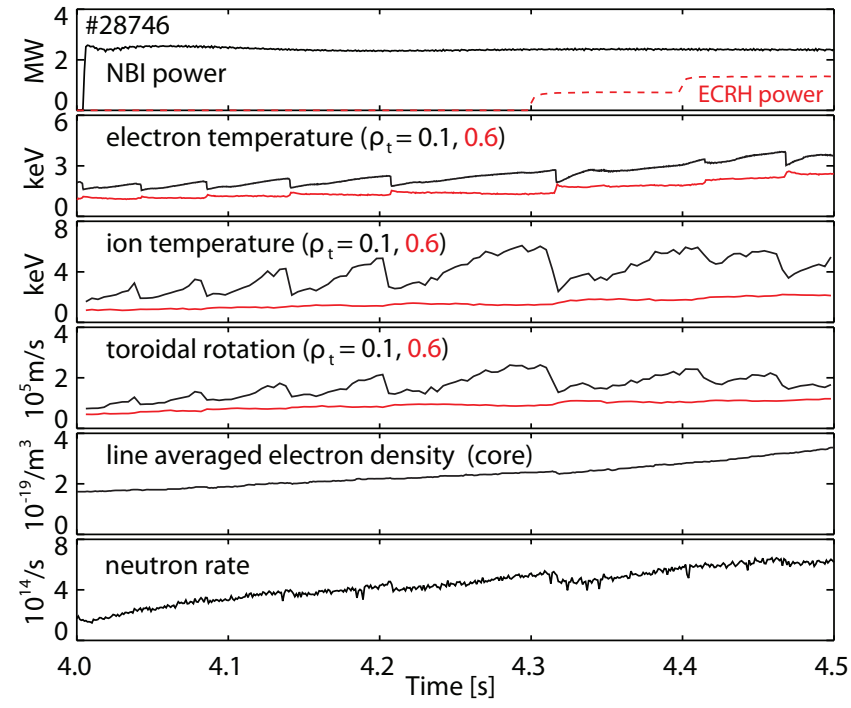

FIG. 1: Characteristic time traces of discharge \#28746. From top to bottom, the ECRH and NBI heating power, the electron temperature, the ion temperature, the toroidal plasma rotation, the line averaged electron density and the neutron rates are shown.

the plasma center, i.e. at the normalized toroidal flux $\rho_{t}=0.1$. The simultaneous increase of the temperature and rotation at $\rho_{t}=0.6$ is evidence of the radial redistribution of heat and momentum due to the crash. The electron density, in contrast, shows only small variations due to its flat profile shape. In the bottom panel of figure 1 , the measured neutron rate, which mainly arises from D-D reactions between fast and thermal ions, is plotted. It shows only a weak decay after the sawtooth crashes.

The fast ions present in discharge \#28746 are generated by the application of $2.5 \mathrm{MW}$ of NBI heating power from a $60 \mathrm{keV}$ source labeled Q3. The geometry of this NBI source is sketched in figure 2 in orange. The source is tilted into the co-current direction and generates a fastion population peaked in the plasma center, as plotted in shades of gray in figure 2 (predicted by the TRANSP code [18]). A theoretical velocity space distribution due to Q3 is depicted in figure $3 \mathrm{a}$ as a function of energy and pitch, with pitch $=v_{\|} / v$ where $v_{\|}$is the fast-ion velocity anti-parallel to the magnetic field. As can be seen in figure 3a, the fast ions from Q3 are injected with a maximum energy of $60 \mathrm{keV}$ and a pitch of $\sim 0.6$ and then diffuse in the velocity space. The gyro radii of these ions are on the order of $\sim 1 \mathrm{~cm}$ and, hence, are very comparable to the normalized fast-ion gyro radii in future fusion devices such as ITER [19].

For the detailed study presented here, 7 toroidal and 7 poloidal LOS, which collect the FIDA radiation along the path of Q3 as shown in figure 2, were connected simultaneously to the FIDA spectrometer. Example spectra measured during $8 \mathrm{~ms}$ long time intervals before (black) and after (red) a sawtooth crash at $t=4.316 \mathrm{~s}$ are shown in figure 4 for two toroidal and two poloidal LOS at dif-
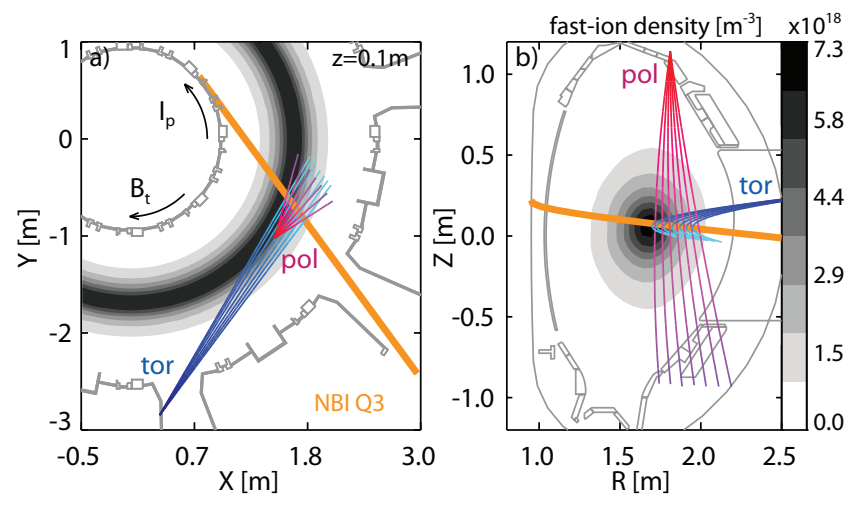

FIG. 2: Top-down (a) and poloidal (b) view of the ASDEX Upgrade tokamak with the path of NBI Q3 in orange. A TRANSP predicted fast-ion distribution generated by NBI Q3 is plotted in shades of gray (\#28746 at $4.3 \mathrm{~s}$ ). In addition, the LOS setup of the FIDA diagnostic is shown. The toroidal LOS are plotted in blue, the poloidal ones in red.

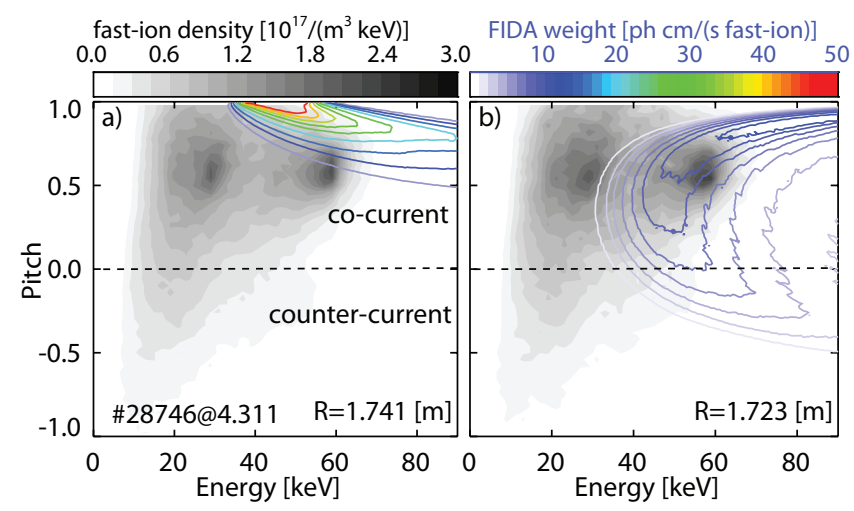

FIG. 3: Theoretical velocity space distribution of fast ions in the plasma center generated by NBI Q3 in \#28746 at $4.3 \mathrm{~s}$, plotted in shades of gray. In colors, weight functions are shown that describe the velocity space observed by a central toroidal (a) and poloidal (b) LOS in the wavelength range between $660 \mathrm{~nm}$ and $661 \mathrm{~nm}$.

ferent radial positions. The spectra can be subdivided from left to right into three distinct parts, as illustrated by the dashed vertical lines in figure 4: Up to $659 \mathrm{~nm}$, the un-shifted D-alpha radiation from the plasma edge $(656.1 \mathrm{~nm})$ and the intense beam and halo radiation are visible [20]. The red-shifted part of the FIDA radiation can be measured in the spectra above $659 \mathrm{~nm}$ and reaches, theoretically, to $661.4 \mathrm{~nm}$ for fast-ion velocity vectors with $60 \mathrm{keV}$ parallel to the LOS. Lastly, at larger wavelengths, the background radiation is visible, consisting of the Bremsstrahlung and weak impurity line emissions. The sawtooth crash causes a significant reduction of the FIDA signal in the plasma center and an increase outside the sawtooth inversion radius (here at $R \sim 1.85 \mathrm{~m}$ ). It is clear that this change of the FIDA radiation is not due to a change of the neutral density along the NBI path, which is responsible for the charge 


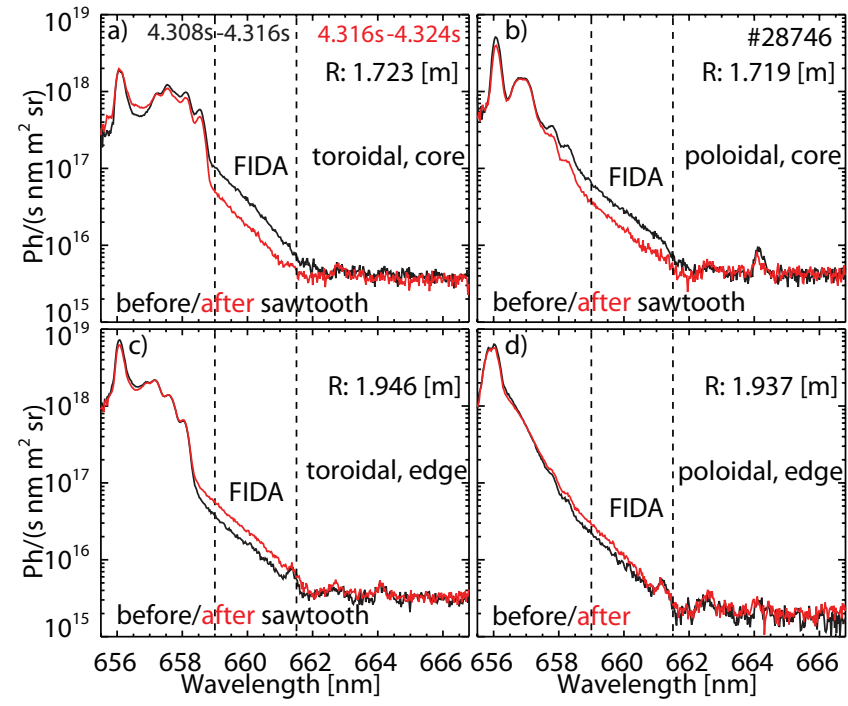

FIG. 4: Spectra from two toroidal (a,c) and two poloidal (b,d) LOS, measured before and after a sawtooth crash in \#28746.

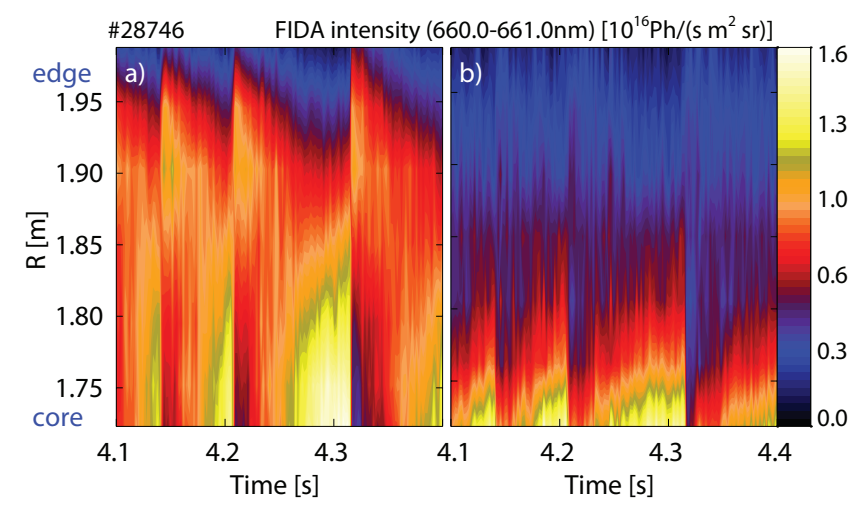

FIG. 5: Evolution of radial FIDA intensity profiles from the toroidal (a) and poloidal LOS (b).

exchange reactions. The neutrals present along the NBI path also emit the beam and halo radiation, which are contained in the spectra as well and are not significantly modified by the crash. Therefore, the measured change of the FIDA signal shows a sawtooth-induced fast-ion redistribution.

The temporal evolution of the FIDA light during the sawtooth crashes is plotted in figure 5 by radial FIDA intensity profiles from the toroidal and poloidal LOS. The profiles are calculated by integrating the spectra between $660 \mathrm{~nm}$ and $661 \mathrm{~nm}$ and by subtracting a constant offset which accounts for the background radiation [20]. The parts of the fast-ion velocity space that are observed in the wavelength interval between $660 \mathrm{~nm}$ and $661 \mathrm{~nm}$ are depicted in figure 3 via the diagnostic weight functions of the toroidal and poloidal LOS. The weight functions describe the probability of fast ions to be neutralized by charge exchange reactions and then to emit D-alpha radiation with certain Doppler shifts towards the FIDA di- agnostic. Their overlap with the fast-ion velocity space distribution is largest for passing fast ions with pitch values close to one for the toroidal LOS and pitch values close to 0.6 for the poloidal LOS. The toroidal profiles in figure 5 , hence, correspond to fast ions that move along the magnetic field lines with energies above $30 \mathrm{keV}$ while the poloidal profiles represent fast ions above $30 \mathrm{keV}$ with similar or larger gyro radii. The temporal evolution of the FIDA signal from the toroidal and poloidal LOS consequently shows that sawteeth substantially change the fast-ion density in both parts of the observed velocity space. After each sawtooth crash, the central FIDA radiation is reduced by about $50 \%$ while it is increased at larger radii.

TRANSP/Kadomtsev - To analyze this measured change quantitatively, forward modeling of the FIDA radiation by the Monte Carlo code FIDASIM [21], which uses theoretical fast-ion distribution functions from the TRANSP code, is applied. TRANSP predicts 4D distribution functions of fast ions and uses the Kadomtsev model for the sawtooth-driven fast-ion redistribution. It assumes complete reconnection of the magnetic field line topology inside the $q=1$ surface during sawteeth and determines the corresponding fast-ion redistribution along the evolving flux surfaces under the assumption of particle conservation. Before sawtooth crashes, the TRANSP-predicted slowing down distribution is peaked in the plasma center, as plotted in shades of gray in figure 2 for discharge $\# 28746$ at $t=4.316 \mathrm{~s}$. After the sawtooth crash, the simulated central fast-ion density is reduced by about $50 \%$ and has a flat profile shape up to the predicted mixing radius at about $2.0 \mathrm{~m}$.

In figures $6 \mathrm{a}$ and $6 \mathrm{~b}$, these theoretical predictions before and after the sawtooth crash at $t=4.316$ s are compared with the FIDA measurements from the toroidal and poloidal LOS, respectively. The experimental data, whose measurement uncertainty is given by error-bars, is shown for two $8 \mathrm{~ms}$ long time intervals before and after the crash. The solid lines in figure 6 correspond to the theoretical fast-ion distribution functions from TRANSP that have been transformed into radial FIDA profiles by FIDASIM. The simulations before (black) and after (red) the sawtooth crash show good agreement with the measurement in both observation geometries, which confirms the predicted redistribution of more than $50 \%$ of the central fast-ion population. The measured and predicted increase of the FIDA radiation outside the sawtooth inversion radius is smaller than the decrease in the center due to volumetric effects in real space. The ions are thrown from small volumes into larger volumes, and thus their density becomes lower.

It should be noted that the simulation yields an upper limit for the fast-ion redistribution caused by the sawtooth induced change of flux surfaces because full reconnection during sawteeth, as assumed by the Kadomtsev model, is typically not the case in our experiment. The standard sawtooth behavior in ASDEX Upgrade is only with partial reconnection [22] and the real changes 


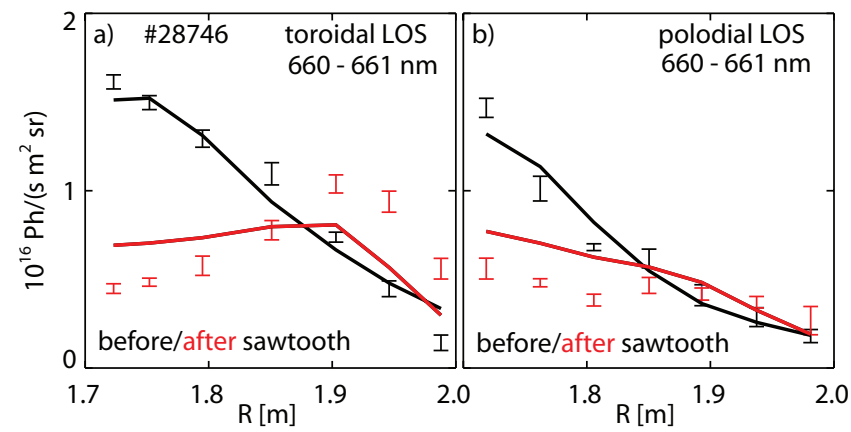

FIG. 6: Radial FIDA intensity profiles from the toroidal (a) and poloidal (b) LOS, measured before and after a sawtooth crash at $t=4.316 \mathrm{~s}$ in discharge \#28746. The simulations from FIDASIM based on TRANSP-predicted fast-ion distribution functions are plotted with solid lines.

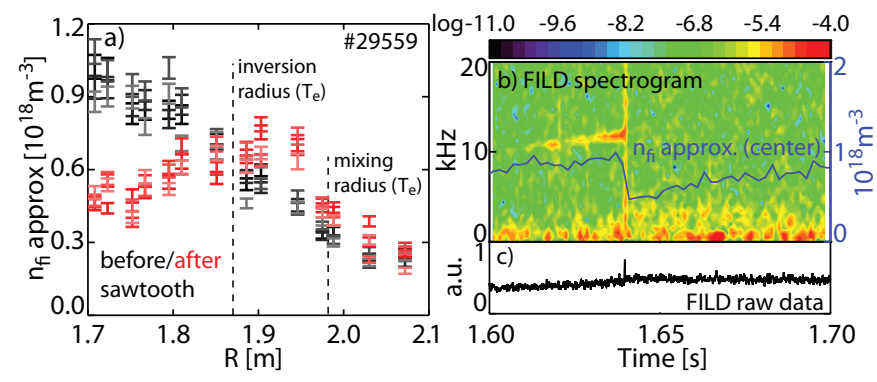

FIG. 7: a) Approximate fast-ion density profiles that represent the velocity space covered by the toroidal LOS and that were measured with $2 \mathrm{~ms}$ exposure time in the presence of a sawtooth crash. b) Spectrogram and raw data (c) from the FILD.

of the field line topology are less than predicted. The Kadomtsev model, therefore, permits us to state that an additional transport mechanism influences the fast ions in our experiment because the simulation shows a smaller redistribution in the fast-ion density than is actually measured. The ExB drift effects, as suggested by [6], can be responsible for this additional fast-ion transport. This effect is supposed to occur in ASDEX Upgrade above a critical fast-ion energy of $50 \mathrm{keV}$. In addition, a sawtooth induced fast-ion redistribution towards more passing orbits could explain the stronger relative change of the FIDA intensity measured by the toroidal LOS outside the sawtooth inversion radius as compared with the poloidal ones. If fast ions are redistributed to more passing orbits, they would reach the part of the velocity space at which the toroidal LOS have their highest measurement probability (see figure 3a). This would also be consistent with the poloidal measurements, which are insensitive to purely passing fast-ions.

Internal fast-ion redistribution - Despite the additional transport effects, the redistributed fast ions remain well confined. The weak changes of the neutron rates in figure 1 already show that sawteeth do not substantially eject fast ions out of the plasma. Otherwise, the neutron
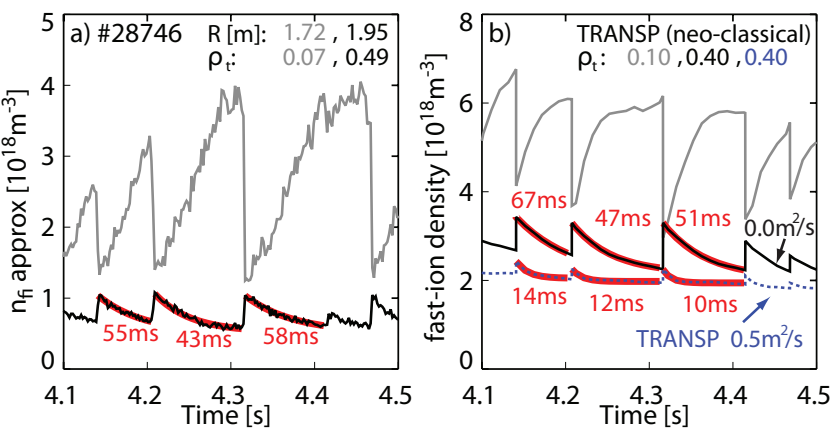

FIG. 8: a) Measured evolution of approximate fast-ion densities that represent the velocity space observed by the toroidal LOS. b) TRANSP simulation of the evolution of the total fast-ion density. The simulation shown in blue represents an anomalous fast-ion transport of $0.5 \mathrm{~m}^{2} / \mathrm{s}$.

rates would drop more significantly. To support this, discharges similar to \#28746 were conducted in which the FILD was operational and in which the FIDA spectrometer was equipped with 15 toroidal LOS to obtain a complete and detailed radial coverage of the plasma. Figure 7a shows approximate fast-ion density profiles inferred from the FIDA measurement for eight time points before and after a sawtooth crash at $1.64 \mathrm{~s}$ in discharge \#29559. The approximate fast-ion densities, $\mathrm{n}_{\mathrm{fi}}$ approx, correspond to the velocity space covered by the toroidal LOS and account for the probability of fast ions to undergo charge-exchange reactions with neutrals and then to emit Balmer alpha radiation. Hereby, the density of neutrals present along the NBI path has been simulated by FIDASIM for each time point and the relative collision energy between fast ions and neutrals has been determined based on the shape of a TRANSP predicted fast-ion velocity distribution. The error-bars plotted in figure 7 a represent the uncertainties from the FIDA measurement. Clearly, the sawtooth-induced changes of the approximate fast-ion densities are outside these measurement uncertainties and indicate an internal fast-ion redistribution that is well described by the inversion and mixing radii derived from the electron temperature measurement. This is supported by the FILD detector, which evidences that the crash does not eject a considerable number of fast-ions. In the raw-data from the FILD, shown in figure $7 \mathrm{c}$, only a small and narrow peak can be observed during the sawtooth crash at $1.64 \mathrm{~s}$. The detector, however, is sensitive enough to detect even weak fast-ions losses, as shown by the spectrogram of the FILD data in figure $7 \mathrm{~b}$. Before the crash, the detector clearly measures fast-ion losses due to a sawtooth precursor mode at $10 \mathrm{kHz}$. These losses do not significantly alter the central fast-ion density, additionally plotted in blue.

Fast-ion transport between sawteeth - The FILD measurement presented in figure $7 \mathrm{c}$ also indicates that the fast ions are well confined in the MHD quiescent phase between the sawteeth. The low level of fast-ion losses after the crash could not be explained if the redistributed 
fast-ions were to quickly diffuse outwards and not remain on their new orbits, e.g. due to micro turbulence [23]. This can be verified thanks to the high temporal resolution of the FIDA diagnostic. Figure $8 \mathrm{a}$ shows the evolution of the approximate fast-ion density in the velocity space observed by two toroidal LOS in discharge \#28746 in the presence of sawteeth. The channel outside of the sawtooth inversion radius exhibits a steep increase when the crashes appear, followed by a slow decay. This decay has been fitted by an exponential function (red) which yields decay times between $50 \mathrm{~ms}$ and $60 \mathrm{~ms}$. These decay times are very well reproduced by a neo-classical simulation of the total fast-ion density from TRANSP, plotted in figure $8 \mathrm{~b}$ for two radial positions. The simulation outside the sawtooth inversion radius, plotted in black, exhibits decay times similar to the measurement. In contrast, when a strong, radially constant anomalous fast-ion diffusion of $0.5 \mathrm{~m}^{2} / \mathrm{s}$ is used in the simulation, as done for the data plotted in blue, significantly shorter decay times are obtained that are even close to those of the electron temperature which are about $5 \mathrm{~ms}$ in this discharge. In addition, the radial profiles, shown in figure 6 , prove the absence of a strong anomalous fast-ion transport. The simulated profiles from the toroidal and poloidal LOS, plotted in black, represent the neo-classical slowing down distribution and agree well with the measurement before the sawtooth crash. When, instead, an anomalous fast-ion transport of $0.5 \mathrm{~m}^{2} / \mathrm{s}$ is applied in the simulation, the modeled profiles have significantly lower values and a flat profile shape. This proves that in the absence of strong MHD activity, the fast ions are well confined under the investigated conditions.

Summary and conclusion - The detailed measurement of the confined fast ions using FIDA spectroscopy evidence a very strong but internal fast-ion redistribution during sawtooth crashes in two different parts of the velocity space. The absence of strong fast-ion losses and weak changes in the measured neutron rates agree well with this observation. The experimental data is in good agreement with theoretical predictions based on TRANSP and the Kadomtsev model but evidences a stronger fast-ion redistribution than predicted. This shows that an additional transport mechanisms, such as ExB drifts, affect the fast ions during sawteeth. Between sawtooth crashes, a classical fast-ion confinement is observed. The temporal evolution, as well as radial profiles of fast-ions agree with neo-classical TRANSP predictions. By extrapolating our results to ITER, the sawtooth induced redistribution of the fast ions with normalized gyro radii similar to the ones at ASDEX Upgrade, will be tolerable with respect to machine safety. The change of the current drive and heating profiles induced by the internal fast-ion redistribution should, however, be taken into consideration.
[1] A. Fasoli, C. Gormenzano, H. Berk, B. Breizman, S. Briguglio, D. Darrow, N. Gorelenkov, W. Heidbrink, A. Jaun, S. Konovalov, R. Nazikian, J.-M. Noterdaeme, S. Sharapov, K. Shinohara, D. Testa, K. Tobita, Y. Todo, G. Vlad, and F. Zonca, Nuclear Fusion 47, S264 (2007).

[2] W. W. Heidbrink, J. M. Park, M. Murakami, C. C. Petty, C. Holcomb, and M. A. Van Zeeland, Phys. Rev. Lett. 103, 175001 (2009).

[3] S. Günter, G. Conway, S. daGraca, H.-U. Fahrbach, C. Forest, M. G. Munoz, T. Hauff, J. Hobirk, V. Igochine, F. Jenko, K. Lackner, P. Lauber, P. McCarthy, M. Maraschek, P. Martin, E. Poli, K. Sassenberg, E. Strumberger, G. Tardini, E. Wolfrum, H. Zohm, and A. U. Team, Nuclear Fusion 47, 920 (2007).

[4] S. von Goeler, W. Stodiek, and N. Sauthoff, Phys. Rev. Lett. 33, 1201 (1974).

[5] F. Porcelli, D. Boucher, and M. N. Rosenbluth, Plasma Physics and Controlled Fusion 38, 2163 (1996).

[6] Y. Kolesnichenko, V. Lutsenko, R. White, and Y. Yakovenko, Nuclear Fusion 40, 1325 (2000).

[7] J. A. Lovberg, W. W. Heidbrink, J. D. Strachan, and V. S. Zaveryaev, Physics of Fluids B: Plasma Physics 1, 874 (1989).

[8] F. B. Marcus, J. M. Adams, A. D. Cheetham, S. Conroy, W. G. F. Core, O. N. Jarvis, M. J. Loughlin, M. Olsson, G. Sadler, P. Smeulders, P. V. Belle, and N. Watkins, Plasma Physics and Controlled Fusion 33, 277 (1991).

[9] B. Stratton, R. Ponck, G. McKee, R. Budny, Z. Chang, F. Wising, and A.Ödblom, Nuclear Fusion 36, 1586 (1996).

[10] F. Marcus, J. Adams, B. Balet, D. Bond, S. Conroy, P.
Howarth, O. Jarvis, M. Loughlin, G. Sadler, P. Smeulders, and N. Watkins, Nuclear Fusion 33, 1325 (1993).

[11] S. Zweben, R. Budny, D. Darrow, S. Medley, R. Nazikian, B. Stratton, E. Synakowski, and G. T. for the TFTR Group, Nuclear Fusion 40, 91 (2000).

[12] W. Heidbrink and G. Sadler, Nuclear Fusion 34, 535 (1994).

[13] G. Sadler, O. Jarvis, P. van Belle, M. Hone, S. Conroy, and J. Adams, Fusion Technology 18, 556 (1990).

[14] S. Nielsen, M. Salewski, H. Bindslev, A. Bürger, V. Furtula, M. Kantor, S. Korsholm, H. Koslowski, A. KrmerFlecken, F. Leipold, F. Meo, P. Michelsen, D. Moseev, J. Oosterbeek, M. Stejner, E. Westerhof, and the TEXTOR team, Nuclear Fusion 51, 063014 (2011).

[15] C. M. Muscatello, W. W. Heidbrink, Y. I. Kolesnichenko, V. V. Lutsenko, M. A. V. Zeeland, and Y. V. Yakovenko, Plasma Physics and Controlled Fusion 54, 025006 (2012).

[16] B. Geiger, R. Dux, R. M. McDermott, S. Potzel, M. Reich, F. Ryter, M. Weiland, D. Wünderlich, M. GarciaMunoz, and the ASDEX Upgrade team, Review of Scientific Instruments 84, 113502 (2013).

[17] M. Garcia-Munoz, H.-U. Fahrbach, H. Zohm, and the ASDEX Upgrade Team, Review of Scientific Instruments 80, 053503 (2009).

[18] A. Pankin, D. McCune, R. Andre, G. Bateman, and A. Kritz, Computer Physics Communications 159, 157 (2004).

[19] M. Shimada, D. Campbell, V. Mukhovatov, M. Fujiwara, N. Kirneva, K. Lackner, M. Nagami, V. Pustovitov, N. Uckan, J. Wesley, N. Asakura, A. Costley, A. Donn, E. 
Doyle, A. Fasoli, C. Gormezano, Y. Gribov, O. Gruber, T. Hender, W. Houlberg, S. Ide, Y. Kamada, A. Leonard, B. Lipschultz, A. Loarte, K. Miyamoto, V. Mukhovatov, T. Osborne, A. Polevoi, and A. Sips, Nuclear Fusion 47, S1 (2007).

[20] B. Geiger, M. Garcia-Munoz, W. W. Heidbrink, R. M. McDermott, G. Tardini, R. Dux, R. Fischer, V. Igochine, and the ASDEX Upgrade Team, Plasma Physics and Controlled Fusion 53, 065010 (2011).
[21] W. Heidbrink, D. Liu, Y. Luo, E. Ruskov, and B. Geiger, Commun. Comput. Phys. 10, 716 (2011).

[22] V. Igochine, O. Dumbrajs, D. Constantinescu, H. Zohm, G. Zvejnieks, and the ASDEX Upgrade Team, Nuclear Fusion 46, 741 (2006).

[23] T. Hauff, M. J. Pueschel, T. Dannert, and F. Jenko, Phys. Rev. Lett. 102, 075004 (2009). 